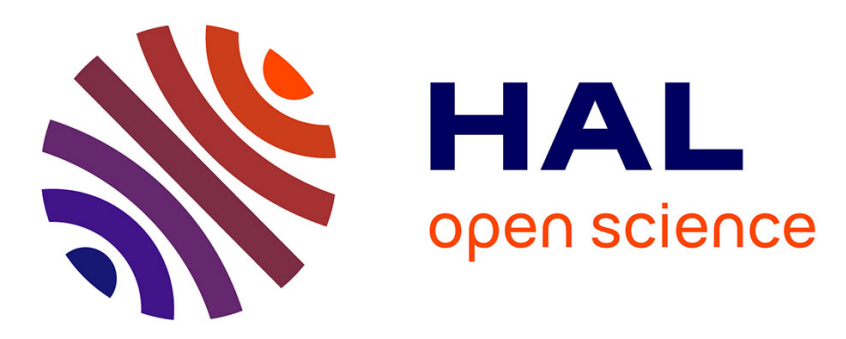

\title{
Small lightning flashes from shallow electrical storms on Jupiter
}

Heidi Becker, James Alexander, Sushil Atreya, Scott Bolton, Martin Brennan, Shannon Brown, Alexandre Guillaume, Tristan Guillot, Andrew Ingersoll, Steven Levin, et al.

\section{To cite this version:}

Heidi Becker, James Alexander, Sushil Atreya, Scott Bolton, Martin Brennan, et al.. Small lightning flashes from shallow electrical storms on Jupiter. Nature, 2020, 584 (7819), pp.55-58. 10.1038/s41586020-2532-1 . hal-03058480

\section{HAL Id: hal-03058480 https://hal.science/hal-03058480}

Submitted on 4 Jan 2021

HAL is a multi-disciplinary open access archive for the deposit and dissemination of scientific research documents, whether they are published or not. The documents may come from teaching and research institutions in France or abroad, or from public or private research centers.
L'archive ouverte pluridisciplinaire HAL, est destinée au dépôt et à la diffusion de documents scientifiques de niveau recherche, publiés ou non, émanant des établissements d'enseignement et de recherche français ou étrangers, des laboratoires publics ou privés. 


\section{Small Jovian lightning flashes indicating shallow electrical storms}

7 Heidi N. Becker ${ }^{*}$, James W. Alexander ${ }^{1}$, Sushil K. Atreya ${ }^{2}$, Scott J. Bolton ${ }^{3}$, Martin J. Brennan ${ }^{1}$,

8 Shannon T. Brown ${ }^{1}$, Alexandre Guillaume ${ }^{1}$, Tristan Guillot ${ }^{4}$, Andrew P. Ingersoll ${ }^{5}$, Steven M.

9 Levin $^{1}$, Jonathan I. Lunine ${ }^{6}$, Yury S. Aglyamov ${ }^{6}$, Paul G. Steffes ${ }^{7}$

109 March 2020

$12{ }^{1}$ Jet Propulsion Laboratory, California Institute of Technology, Pasadena, CA, USA

$13{ }^{2}$ Climate and Space Sciences and Engineering, University of Michigan, Ann Arbor, MI, USA

$14{ }^{3}$ Southwest Research Institute, San Antonio, TX, USA

$15{ }^{4}$ Université Côte d'Azur, OCA, Lagrange CNRS, Nice, France

$16{ }^{5}$ California Institute of Technology, Pasadena, CA USA

$17{ }^{6}$ Cornell University, Ithaca, NY USA

$18{ }^{7}$ School of Electrical and Computer Engineering, Georgia Institute of Technology, Atlanta, GA, 19 USA

20 Corresponding author email: Heidi.N.Becker@jpl.nasa.gov

21 
Jovian lightning flashes were characterized by a number of missions that visited Jupiter over the past several decades. Imagery from the Voyager 1 and Galileo spacecraft led to a flash rate estimate of $\sim 4 \times 10^{-3}$ flashes $/ \mathrm{km}^{2} / \mathrm{yr}$ on Jupiter. ${ }^{1,2}$ The spatial extent of Voyager flashes was estimated to be $\sim 30 \mathrm{~km}$ at half-width half-maximum intensity (HWHM), but the camera was unlikely to have detected the dim outer edges of the flashes given weak response to the brightest spectral line of Jovian lightning emission, the $656.3 \mathrm{~nm} \mathrm{H}$-alpha line of atomic hydrogen (known from lab experiments). ${ }^{1,3-6}$ The spatial resolution of Galileo and New Horizons cameras allowed investigators to confirm twenty-two flashes with HWHM >42 km and estimate one between $37-45 \mathrm{~km} .{ }^{1,7,8,9}$ These flashes, with optical energies only comparable to terrestrial "superbolts" $\left(2 \times 10^{8}-1.6 \times 10^{10} \mathrm{Joules}\right)$, have historically been interpreted as tracers of moist convection originating near the 5 bar level of Jupiter's atmosphere (assuming photon scattering from points beneath the clouds). ${ }^{1-}$ 3,7,8,10-12 All previous optical observations of Jovian lightning have been limited by camera sensitivity, proximity to Jupiter, and long exposures ( $680 \mathrm{~ms}$ to $85 \mathrm{~s}$ ) hence some measurements were likely superimposed flashes reported as one., ${ }^{1,2,7,9,10,13}$ Here we report

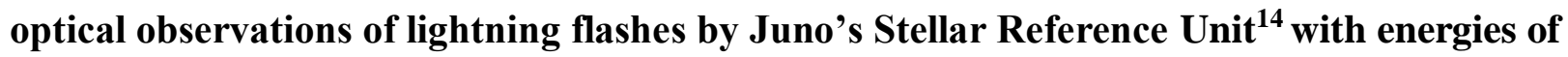
$\sim 1^{5}-10^{8}$ Joules, flash durations as short as $5.4 \mathrm{~ms}$, and inter-flash separations of tens of milliseconds. The observations exposed Jovian flashes with typical terrestrial energies. The flash rate is $\sim 6.1 \times 10^{-2}$ flashes $/ \mathrm{km}^{2} / \mathrm{yr}$, more than an order of magnitude greater than hitherto seen. Several flashes are of such small spatial extent they must originate above the 2 bar level, where there is no liquid water. ${ }^{15,16}$

Juno's Stellar Reference Unit (SRU) captured images of Jovian lightning on the dark side of Jupiter from a unique perspective of as close as 53,000 km above the 1 bar level $(30 \mathrm{~km} / \mathrm{pixel}$ resolution). The SRU is a broadband (450-1100 nm) imager designed to detect dim stars in support of spacecraft attitude determination. The camera's point spread function (PSF) spreads the optical signal of a point source over $\sim 5 \times 5$ pixels, allowing unambiguous identification of small optical sources (see Extended Data Fig. 1). Fourteen lightning flashes (see Extended Data Table 1) were observed between $\sim 46^{\circ} \mathrm{N}$ and $75^{\circ} \mathrm{N}$, and one at $51^{\circ} \mathrm{S}$, (planetocentric) during a 9image lightning survey between perijoves 11 (7 February 2018) and 17 (21 December 2018) that covered $1 \times 10^{10} \mathrm{~km}^{2}$ of the planet. The storms observed in motion compensated images (such as Figure 1 panel a) were confirmed to appear in cyclonic "belt" regions, consistent with prior 
optical observations of Jovian lightning by Galileo ${ }^{1}$. Juno's Microwave Radiometer ${ }^{17}$ (MWR) provided supporting microwave observations, detecting lightning ${ }^{18}$ within $\sim 0.4$ seconds to 3 minutes of each SRU detection in the Northern hemisphere while its $600-\mathrm{MHz}$ beam covered the SRU flash location (see Extended Data Fig. 2 and Supplementary Data 1).

The novel combination of close observation distance, camera sensitivity, and the spin of the Juno spacecraft (two revolutions per minute) enabled lightning detection at higher resolution than previously possible. Two $1 \mathrm{~s}$ SRU exposures were collected without motion compensation (hereafter "NoTDI") allowing the camera's field of regard to be panned across Jupiter at a rate of 1 pixel every $2.7 \mathrm{~ms}$. A dim "string of pearls" trail of multiple spots along the scan direction was observed in one of these images (Figure 1 panel b), assumed to be flashes from the same storm location. Inter-flash separations of only tens of milliseconds, and flash durations as short as 5.4 $\mathrm{ms}$, were deduced. The global flash rate estimate based on the NoTDI imagery is $6.1 \times 10^{-2}$ flashes $\mathrm{km}^{-2}$ year $^{-1}$, 15 times higher than previous estimates derived from optical observations. ${ }^{1,2}$

The Juno observations have unveiled Jovian lightning flashes with optical energies similar to typical terrestrial flashes $\left(1 \times 10^{5}-2 \times 10^{7} \mathrm{~J}\right) .{ }^{19}$ Figure 2 illustrates that the optical energies of the SRU flashes extend far below the $2 \times 10^{8}-1.6 \times 10^{10} \mathrm{~J}$ range that was detectable in prior broadband surveys. ${ }^{1,2,7}$ Although optical energy is just part of the total energy generated by lightning (for example, $5-18 \mathrm{kHz}$ radio emissions from terrestrial lightning range from 10 to $10^{8}$ $\mathrm{J}$, where strokes above $10^{6} \mathrm{~J}$ are considered "superbolts" ${ }^{20}$ ), optical detection of lightning in this new regime, and the higher flash rate suggested for it, provides important constraints for understanding energy dissipation rates in Jupiter's atmosphere and reduces the reliance on terrestrial-based assumptions where data are lacking. ${ }^{2,11}$

We identified lightning flashes with estimated widths as small as $\sim 33 \mathrm{~km}$ and as large as $250 \mathrm{~km}$. It is customary to use the half-width at half-maximum (HWHM) of a lightning flash (the radial distance over which the flash radiance drops to half the peak value) to infer the lightning's depth within the atmosphere. ${ }^{1,3,7,8}$ The deeper the origin of the flash photons, the more they will expand radially outward as they scatter through the atmosphere before being released to space, and the larger the observed HWHM. The HWHM was estimated for six flashes where the de-convolved brightness distribution had sufficient symmetry and simplicity to make a reasonable estimate (see Extended Data Fig. 3). These are estimated maxima as we could not confirm sub-pixel values; 
many are the half-width value and the actual HWHM is suspected to be smaller. We estimated SRU flash depths based on a radiative transfer model where depth $=1.5 \times$ HWHM and the top scattering layer is at the 0.14 bar level $^{1}\left(40 \mathrm{~km}\right.$ above the $\left.1 \mathrm{bar} \mathrm{level}^{15}\right)$. At this "top" scattering level ammonia ice crystals would likely be the predominant scattering particles although water ice particles might also be carried up to this level by strong updrafts. ${ }^{12,21}$ The pressuretemperature-depth data from the Galileo probe $^{15}$ provided the pressure levels corresponding to the computed depths below the top scattering layer.

Four of the SRU flashes originate between 1.4 and 1.9 bars (inset Figure 3), hereafter "shallow lightning," which is a surprising result given that conventional models of lightning generation by charge separation require both liquid and solid condensate. ${ }^{10}$ Although intense storms are expected to loft water-ice crystals to such heights, ${ }^{21}$ no liquid water can exist at these altitudes as temperatures above the 2 bar level are below $-66^{\circ} \mathrm{C},{ }^{15}$ which is well below a plausible minimum temperature for supercooled water $\left(-40^{\circ} \mathrm{C}\right)^{16}$. If a non-inductive charge separation mechanism involving mixed-phase clouds is responsible for the shallow lightning, then another source of liquid is required. The possible role of ammonia is shown conceptually in Figure 3. Should water ice particles from below be lifted by very strong updrafts to altitudes between 1.1 and 1.5 bars, equilibrium thermodynamics predicts that they can adsorb ambient ammonia vapor and create a mixed ammonia-water $\left(\mathrm{NH}_{3}-\mathrm{H}_{2} \mathrm{O}\right)$ liquid. ${ }^{22,23,24}$ The collision of this falling liquid with upward moving water-ice particles, or falling ice crystals with lower terminal velocities, would constitute a mixed phase liquid- and solid-bearing cloud in which charge transfer, charge separation, and cloud electrification might occur (see Methods). This would be unlike any process which occurs on Earth, but it is enabled by the large presence of ammonia at Jupiter which acts as a strong $\mathrm{H}_{2} \mathrm{O}$ antifreeze at the extremely low temperatures in the 1.1-1.5 bar region.

Alternatively, Juno's SRU may have observed lightning generated without any liquid. On Earth, lightning flashes have been observed to originate in cloud anvils ("cumulonimbus incus") at altitudes above $\sim 10 \mathrm{~km}$ (below $-45^{\circ} \mathrm{C}$ ) and significant charging was inferred to have occurred locally within the anvil. ${ }^{25}$ Recent airborne measurements have confirmed that charge separation can occur in the extremely dynamic environment inside anvils, without any detected supercooled liquid water, due to the collision and transfer of charge between ice particles of broadly differing size. ${ }^{26,27}$ Anvils have been predicted to form above the 2 bar level at Jupiter ${ }^{28}$, and modeling has 
114

115

116 117 within anvils (see Methods).

118

119

120

121

122

123

124

125

126

127

128

129

130

131

132

133

134

135

136

137

138

139

140

141

142 imagery. ${ }^{1,12}$ meteorological significance. using Juno instrumentation.

shown that updrafts in Jovian thunderclouds can loft water ice particles of different sizes up to the 1 bar level. ${ }^{21}$ It is therefore plausible that shallow Jovian lightning is the result of noninductive charge separation from ice-ice collisions in high reaching cumulonimbus clouds or

Finally, it is possible that local cloud clearings could allow flashes originating in the water cloud to appear small due to reduced scattering by a lower cloud top. We argue that the small flash located above the elevated cloud in the moonlit SRU image (lower flash in Figure 1 panel a) is evidence against a lower cloud top explanation. This flash is also consistent with prior observations that linked lighting flashes to local elevated cloud structures in dayside or moonlit

The remaining SRU flashes originate in the liquid water cloud layer (deeper than the 3 bar level), suggesting that the more familiar liquid water and water-ice electrification mechanism is also occurring at greater depths. The more poleward latitudes of the shallow lightning are noted (see inset Figure 3), however the existing sample set is too small as of yet to infer any latitudinal

Although some SRU detections are consistent with the conventional theory of lightning originating in regions of mixed-phase water condensation, the presence of shallow lightning implied by the Juno observations requires that we consider the possibility of multiple mechanisms for generating lightning in different pressure-temperature environments. By extension, multiple shallow lightning events would suggest that strong localized updrafts are frequent events in Jupiter's atmosphere. Because ammonia is only visible in the microwave when in vapor form, an ammonia-water liquid created by adsorption of ammonia vapor onto water ice particles may partially explain the decades long mystery of observed ammonia depletion in Jupiter's atmosphere. ${ }^{29-31}$ Continued observations by Juno's SRU are anticipated to increase our knowledge of the occurrence rate of shallow lightning with latitude, providing an important component of a broader effort to understand Jupiter's atmospheric convection and composition 
143

144

145

146

147

148

149

150

151

152

153

154

155

156

157

158

159

160

161

162

163

164

165

166

167

168

\section{References}

1. Little, B. et al. Galileo images of lightning on Jupiter. Icarus 142, 306-323 (1999).

2. Borucki, W.J., Bar-Nun, A., Scarf, F.L., Cook II, A.F., \& Hunt, G.E. Lightning activity on Jupiter. Icarus 52, 492-502 (1982).

3. Borucki, W.J. \& Williams, M.A. Lightning in the jovian water cloud. J. Geophys. Res. D9 91, 9893-9903 (1986).

4. Danielson, G.E., Kupferman, P.N., Johnson, T.V., and Soderblom, L.A. Radiometric performance of the Voyager cameras. Journal of Geophysical Research, 86, A10, 8683-8689 (1981).

5. Smith, B.A., et al., Voyager imaging experiment. Space Science Reviews, 21 103-127 (1977).

6. Borucki, W.J., McKay, C.P., Jebens, D., Lakkaraju, H.S., Vanajakshi, C.T. Spectral irradiance measurements of simulated lightning in planetary atmospheres. Icarus 123, 336-344 (1996).

7. Baines, K. H. et al. Polar lightning and decadal-scale cloud variability on Jupiter. Science 318, 226-229 (2007).

8. Dyudina, U.A., Ingersoll, A.P., Vasavada, A.R., Ewald, S.P., and the Galileo SSI Team. Monte carlo radiative transfer modeling of lightning observed in Galileo images of Jupiter. Icarus 160, 336-349 (2002).

9. Dyudina, U.A. et al. Lightning on Jupiter observed in the $\mathrm{H} \alpha$ line by the Cassini imaging science subsystem. Icarus 172, 24-36 (2004).

10. Yair, Y., Levin, Z., \& Tzivion, S. Lightning generation in a Jovian thundercloud: results from an axisymmetric numerical cloud model. Icarus 115, 421-434 (1995).

11. Rinnert, K. Lightning on other planets. J. Geophys. Res. D4 90, 6225-6237 (1985).

12. Gierasch, P.J. et al. Observations of moist convection in Jupiter's atmosphere. Nature 403, 628-630 (2000).

13. Borucki, W.J. \& Magalhaes, J.A. Analysis of Voyager 2 images of Jovian lighting. Icarus 96, 1-14 (1992). 
169 14. Becker, H.N. et al. The Juno radiation monitoring (RM) investigation. Space Sci. Rev., 213, $170 \quad 507-545$ (2017).

171 15. Seiff, A., et al. Thermal structure of Jupiter's atmosphere near the edge of a 5- $\mu \mathrm{m}$ hot spot in 172 the north equatorial belt. Journal of Geophysical Research, 103, E10, 22,857-22,889 (1998).

173 16. Pruppacher, H.R., Klett, J.D., Microphysics of Clouds and Precipitation. (Kluwer Academic 174 Publishers, Dordrecht, ed. 2, 1997).

175 17. Janssen, M.A. et al. MWR: microwave radiometer for the Juno mission to Jupiter. Space Sci. 176 Rev. 213, 139-185 (2017).

177 18. Brown, S. et al. Prevalent lightning sferics at 600 megahertz near Jupiter's poles. Nature, $178 \quad 558,87-90(2018)$.

179 19. Turman, B.N. Detection of lightning superbolts. Journal of Geophysical Research 82, 25661802568 (1977).

181 20. Holzworth, R.H., McCarthy, M.P, Brundell, J.B., Jacobson, A.R. \& Rodger, C.J. Global 182 distribution of superbolts. Journal of Geophysical Research: Atmospheres 124, 9996-10,005 183 (2019).

184 21. Yair, Y., Levin, Z. \& Tzivion, S. Microphysical processes and dynamics of a Jovian 185 thundercloud. Icarus 114, 278-299 (1995).

186 22. Lewis, J.S. The clouds of Jupiter and the NH3.H2O and NH3.H2S systems. Icarus 10, $365-$ 187378 (1969).

188 23. Weidenschilling, S.J. and Lewis, J.S. Atmospheric and cloud structures of the Jovian planets. 189 Icarus 20, 465-476 (1973).

190 24. Guillot, T., Stevenson, D.J., Atreya, S.K., Bolton, S.J., \& Becker, H.N. Storms and the 191 depletion of ammonia in Jupiter: I. microphysics of "mushballs.” Preprint at 192 https://www.essoar.org/doi/abs/10.1002/essoar.10502154.1 (2020).

193 25. Kuhlman, K.M., MacGorman, D.R., Biggerstaff, M.I., \& Krehbiel, P.R. Lightning initiation 194 in the anvils of two supercell storms. Geophysical Research Letters 36, L07802 (2009). 
208

209

210

26. Dye, J.E. \& Bansemer, A. Electrification in mesoscale updrafts of deep stratiform and anvil clouds in Florida. Journal of Geophysical Research: Atmospheres 124, 1021-1049 (2019).

27. Dye, J.E. et al. Electric fields, cloud microphysics, and reflectivity in anvils of Florida thunderstorms. Journal of Geophysical Research 112, D11215, doi:10.1029/2006JD007550 (2007).

28. Stoker, C.R. Moist convection: a mechanism for producing the vertical structure of the jovian equatorial plumes. Icarus 67, 106-125 (1986).

29. Li, C., et al. The distribution of ammonia on Jupiter from a preliminary inversion of Juno microwave radiometer data. Geophysical Research Letters 44, 5317-5325 (2017).

30. Bolton, S.J., et al. Jupiter's interior and deep atmosphere: The initial pole-to-pole passes with the Juno spacecraft. Science 356, 821-825 (2017).

31. de Pater, I. Jupiter's zone-belt structure at radio wavelengths II. Comparison of observations with model atmosphere calculations. Icarus 68, 344-365 (1986).

Fig. 1 | Images from Juno SRU Jovian lightning survey. Scale bars show pixel signal levels in analog-to-digital units. Background signal has been subtracted. a. SRU image 12 from perijove 11, collected using image motion compensation. Yellow arrows point to flashes of Jovian lightning on Jupiter's dark side. The dim "tail" below the top flash is an artifact of the motion compensation. Insets are magnified views of these small signatures which are spread out by the camera's point spread function. Cloud top illumination is due to moonlight from Jupiter's moon, Io. b. Scanned SRU image 12 from perijove 14 ("NoTDI" image \#2), collected without motion compensation. A dim "string of pearls" trail of multiple flashes from the same storm location is seen in the along-scan direction, as well as an adjacent "neighbor" flash observed 258-13,800 km away depending on when the flashes occurred during the scan. "String of pearls" flashes are numbered 1-4 with inter-flash time separations shown in the magnified view of the region (Flash 3 may be three separate events, with time separations so indicated). Latitudes grids shown in the background correspond to the start of the image scan. 
223

224

225

226

227

228

229

230

231

232

233

234

235

236

237

238

239

240

241

242

243

244

245

246

247

248

Fig. 2 | Optical energies of lightning flashes observed by the Juno SRU and past broadband visible imagers. The flash energy data from each instrument are plotted as a cumulative frequency distribution in order of increasing optical energy. Galileo's Storm 10 observations are plotted separately from Storms $7 \& 8$ due to the difficulty encountered by Galileo investigators in distinguishing flashes from Storm 7 from those of Storm 8. ${ }^{1}$ SRU flash energies are shown for computations which treat each flash as a patch of light on a Lambertian surface (green stars) and as a point source (blue stars). The SRU cumulative frequency distributions have been shifted up by a factor of ten to highlight the lower optical energies detectable by the SRU. Optical energies of terrestrial flashes and "superbolts"19 are indicated by grey bars.

\section{Fig. 3 | Conceptual illustration of lightning generation above and below Jupiter's 3 bar}

level. Energetic updrafts (black arrows) loft water ice particles to altitudes between 1.1 and 1.5 bars where adsorption of ammonia gas onto ice particles melts the ice, creating falling liquid ammonia-water $\left(\mathrm{NH}_{3}-\mathrm{H}_{2} \mathrm{O}\right)$ particles (green drops). Charge separation occurs as the $\mathrm{NH}_{3}-\mathrm{H}_{2} \mathrm{O}$ particles collide with upward moving water-ice, followed by lightning. At pressures greater than $\sim 3$ bars, temperatures are above the limit for supercooled water (white line, $\sim 233 \mathrm{~K}$ ) and lightning is generated in pure water clouds. Radial half-width at half-maximum intensity distances, estimated maximum depths of origin (pressure levels), and latitudes of observed SRU lightning flashes are shown (inset). 


\section{Methods}

Instrument properties. The SRU is a visible imager with a 16.4 degree square field of view, a $29.924 \mathrm{~mm}$ focal length, and spatial resolution of $0.57 \mathrm{mrad}$ per pixel. The camera utilizes a frame transfer silicon CCD with a 17 micron pixel pitch and a 512 x 512 pixel imaging region. ${ }^{14}$ No filters are used in the optical system.

Camera sensitivity. We used the radiometric calibration method that was used for the Galileo solid-state imaging camera ${ }^{32}$ to calculate SRU camera pixel response in units of output analog-todigital data number (DN) pixel $^{-1} \mathrm{~s}^{-1}$ in response to a spectrally neutral scene radiance (having equal energy at each wavelength, and the units $\left.\mathrm{W} \mathrm{cm}^{-2} \mathrm{sr}^{-1} \mathrm{~nm}^{-1}\right)$.

$$
\text { camera pixel response }=\frac{A_{o} a_{i j}}{f^{2} C} \int \frac{Q T(\lambda) I \lambda}{h c} d \lambda
$$

$A_{o}$ is the $4.155 \mathrm{~cm}^{2}$ collecting area of the optics, $a_{i j}$ is the area of a CCD pixel $\left(2.89 \times 10^{-6} \mathrm{~cm}^{2}\right)$, $f$ is the camera focal length, $C$ is the SRU camera gain of 15.47 signal electrons/DN, $Q T$ is the total throughput of the SRU optical system (the CCD quantum efficiency $\times$ the optics transmission, shown as a function of wavelength in Extended Data Fig. 1), $I$ is the scene radiance (assumed to be constant), $h$ is the Planck constant, $c$ is the speed of light, and $\lambda$ is wavelength. The integration was performed over the SRU bandpass (450 to $1100 \mathrm{~nm}$ ), and solved using a $1000 \mathrm{DN}_{\text {pixel }}{ }^{-1} \mathrm{~s}^{-1}$ response assumption. The SRU camera sensitivity is $3.346 \times 10^{13} \mathrm{DN} / \mathrm{W}$ $\mathrm{cm}^{-2} \mathrm{sr}^{-1} \mathrm{~nm}^{-1}$ (per pixel, per second), and the energy incident on the collecting area of the optics is $2.6 \times 10^{-17} \mathrm{~J}$ per DN. We repeated the calibration using simulated Jovian lightning spectra at 1 bar and 5 bars $^{6}$ which yielded lower camera responses of $2.683 \times 10^{13}$ and $2.297 \times 10^{13} \mathrm{DN} / \mathrm{W}$ $\mathrm{cm}^{-2} \mathrm{sr}^{-1} \mathrm{~nm}^{-1}$ (per pixel, per second), respectively. The greater dominance of the $656.28 \mathrm{~nm} \mathrm{H \alpha}$ line in the 1 bar spectrum is responsible for the differences. The two simulated spectra address pressure levels near the general regions of our inferred flash origins, but they are not exceedingly different from the neutral spectrum result. We used the neutral spectrum result for the flash energy calculations reported herein in order to be consistent with the approach used by the Galileo investigators ${ }^{1}$ and to allow direct comparison to the Galileo data.

Lightning flash identification. Raw pixel data for the nine SRU lightning survey images are provided in Supplementary Data 2. To supplement visual inspection an automated search was 
278 performed on the images to locate candidate lightning signatures. The approach was similar to

279 the method used by Juno's Radiation Monitoring Investigation to identify bright radiation

280

281

282

283

284

285

286

287

288

289

290

291

292

293

294

295

296

297

298

299

300

301

302

303

304

305

306

307

308

signatures in SRU images. ${ }^{14}$ Each pixel was assessed to determine whether its signal was a local maximum in the $5 \times 5$ pixel region around it. If so, its signal was further assessed to determine whether it was brighter than all eight of the immediately adjacent pixels by a threshold amount ("Th"). ${ }^{14}$ A threshold of 30 DN above the local background was generally used. When dimmer flashes were suspected (the faint "string of pearls" flashes in NoTDI image \#2) the threshold was dropped to $15 \mathrm{DN}$. Thresholds were at least five times the noise of the local background level, depending on the level of signal from moonlit clouds or scattered sunlight. To accommodate larger lightning flashes which did not meet the criterion for all eight immediate neighbors, a $7 \times$ 7 pixel region around the pixel was assessed. The criterion for this assessment was that the pixel be $>$ Th above at least 18 pixels in the region, or $>$ Th above all sixteen of its second-nearest neighbors, to be identified as a candidate. ${ }^{14}$ In practice, all of the lightning signatures that were found by the automated search were also easily identified using visual inspection. Visual inspection was the final deciding factor in confirming lightning signatures. Following thresholding, an $11 \times 11$ pixel window was placed around the brightest pixel of each identified signature and the median value of each border was calculated. The process was repeated for the inner $9 \times 9$, and $7 \times 7$, pixel windows about the brightest pixel. The minimum of the 12 median values was taken as the sum of the local background level plus the electronic offset level ( $\sim 545$ DN for SRU-1, the SRU unit used for the survey) and was subtracted from the raw values of all pixels in the region local to the candidate storm (hereafter referred to as "background subtraction"). The sum of all background-subtracted pixel values from the flash signature is the "DNsum" used to estimate flash optical energy. Background-subtracted signature morphology was visually inspected to exclude ionizing radiation signatures from the lightning flash candidate list. Extended Data Fig. 4 illustrates the morphology differences between signals from optical sources and ionizing particles. Lightning could be differentiated from penetrating radiation signatures as the later have asymmetric morphologies which fall off abruptly to background levels and do not follow the energy distribution of the camera PSF (the appearance of penetrating particle signatures is not influenced by the optics).

Background subtraction for flash 13_12_0 was based on visual inspection of the image and the raw pixel array values, due to the complexity of auroral emission local to the flash. An additional 
step was taken prior to thresholding and background subtraction for NoTDI Image \#2. Hot pixels are pixels with atypically high signal rates due to defects acquired during fabrication or radiation exposure. The expected hot pixel signal levels following a $1 \mathrm{~s}$ exposure were subtracted from the known hot pixel locations to aid clean assessment of the relatively dim "string of pearls" signatures. The mapping of the hot pixel signal rates (per second) is provided in Supplementary Data 3.

Mapping flash locations on Jupiter. To determine the locations of the lightning flashes on Jupiter, we first computed the centroid of each identified lightning flash ( $\left.\mathrm{x}_{\text {cent }}, \mathrm{y}_{\text {cent }}\right)$ in pixel units, with array pixels numbered 1 to 512 (Extended Data Table 2). The coordinate system origin was placed at the center of pixel $(1,1)$. The flash centroid was then converted to the 0 to 511 pixel coordinate system of the SRU (instrument) frame, which has a boresight coordinate $(255.5,255.5)$ and a reversed y-axis numbering scheme (see Extended Data Fig. 5). We then used the following approach ${ }^{14}$ to convert the centroid components into a unit vector, correct for the camera's optical distortion, and transform the unit vector into the spacecraft frame in J2000 at the start of the image exposure time (time " $t$ "). The intercept of this vector on Jupiter is the flash location.

The centroid measurements define a vector in the SRU frame. The tangents $\left(\tan _{x r e f}, \tan _{y r e f}\right)$ of this vector, projected onto the focal plane, are computed by

$$
\tan _{x r e f}=\frac{x_{c e n t}-255.5}{f l_{\text {ref }}}, \quad \tan _{y r e f}=\frac{y_{\text {cent }}-255.5}{f l_{\text {ref }}}
$$

where $f l_{r e f}=1760.21137$ pixels (the focal length of the camera expressed in pixel units).

The position $\left(\tan _{x r e f}, \tan _{y r e f}\right)$ is radially corrected for optical distortion using following formulae:

$$
R=\left(\tan _{x r e f}^{2}+\tan _{y r e f}^{2}\right)^{1 / 2} \text { and } \mathrm{f}(R)=\mathrm{a}_{0}+\mathrm{a}_{1} R+\mathrm{a}_{2} \mathrm{R}^{2}+\mathrm{a}_{3} \mathrm{R}^{4}
$$

where $\mathrm{a}_{0}=0.999432579, \mathrm{a}_{1}=-0.0295412410, \mathrm{a}_{2}=0.2733020107$, and $\mathrm{a}_{3}=-1.9368112951 . \mathrm{f}(R)$ is the radial correction factor, and $\left(\mathrm{t}_{\mathrm{x}}, \mathrm{t}_{\mathrm{y}}\right)$ is the optical distortion corrected position of the measurement in the image plane computed by $\left(\mathrm{t}_{\mathrm{x}}, \mathrm{t}_{\mathrm{y}}\right)=\mathrm{f}(R)\left(\tan _{x r e f}, \tan _{\mathrm{yref}}\right)$.

The unit vector of the measurement in the SRU (instrument) frame is given by

$$
\boldsymbol{V}_{S R U}=\frac{\left(1,-t_{x},-t_{y}\right)}{\left(1+t_{x}^{2}+t_{y}^{2}\right)}
$$


and the calibrated transformation $\boldsymbol{T}_{S R U_{-} t o_{-} S C}$, maps the SRU measurement vector $\boldsymbol{V}_{S R U}$ to a Juno spacecraft frame pointing vector $\boldsymbol{V}_{S C}$ by

$$
\boldsymbol{T}_{S R U_{-} t t_{-} S C} \boldsymbol{V}_{S R U}=\boldsymbol{V}_{S C}
$$

with

$$
\boldsymbol{T}_{S R U_{-} t o_{-} S C}=\left[\begin{array}{ccl}
0.420419 & 0.90732884 & 0.001389 \\
-0.000795 & -0.000795 & 1.0 \\
0.907330 & -0.42042011 & 0.000233
\end{array}\right]
$$

The time dependent transformation from the Juno Spacecraft frame to $\mathrm{J} 2000$ at ephemeris time $\mathrm{t}$ is $\boldsymbol{T}_{S C_{-} t t_{-} J 2000}(\mathbf{t})$, determined using the Juno SPICE kernels, ${ }^{33}$ which compute the position and attitude of Juno. The SRU measurement in $\mathrm{J} 2000, \boldsymbol{V}_{\mathrm{J} 2000}$, is given by

$$
\boldsymbol{V}_{\mathrm{J} 2000}=\boldsymbol{T}_{S C_{\_} t t_{-} J 2000} \boldsymbol{V}_{S C}=\boldsymbol{T}_{S C_{-} t t_{-} J 2000} \boldsymbol{T}_{S R U_{-} t t_{-} S C} \boldsymbol{V}_{S R U}
$$

The CCD x-axis (along-row direction) and y-axis (along-column direction) are designated "Y" and " $Z$ ", respectively, in the Juno frame kernel ${ }^{33}$, where " $X$ " is the SRU boresight vector. We mapped the planetocentric latitude and System III West longitude of each flash and computed Juno's range to the flash location making standard SPICE Toolkit ${ }^{34}$ corrections for one-way light time and stellar aberration. Pixel resolutions shown in Extended Data Table 1 include correction for optical distortion at the flash location in the image plane and represent the average of the along-row and along-column pixel dimensions (which differed by no more than one percent). We do not have knowledge of when the flashes occurred during the NoTDI image scans, therefore the lack of motion compensation introduces uncertainty as to their location. To bound the range of possible storm locations for these flash centroids, the computations were repeated for the spacecraft geometry corresponding to the end of the exposure time.

Flash deconvolution. To estimate the true profiles of the SRU flashes (prior to having been convolved with the camera PSF), background-subtracted pixel windows containing the flash signatures were de-convolved using MATLAB's Richardson-Lucy iterative restoration algorithm, deconvlucy ${ }^{35}$. The energy distribution shown in Extended Data Fig. 1 was used as the input PSF. The "readout" parameter (variance of the camera read noise; read noise squared) was set to 9. SRU CCD read noise is $\sim 3 \mathrm{DN}$ (48 signal electrons, rms), and dark current is negligible at the $-40^{\circ} \mathrm{C}$ flight temperature. Photoresponse non-uniformity is $0.02,1$-sigma. Background 
subtraction produced small negative values (close to the camera noise) in some pixels surrounding the flash area. These negative values were set to zero prior to deconvolution. Three hundred to one thousand iterations were used, which converged all solutions. Extended Data Fig. 6 shows an example reconstruction.

"NoTDI" image \#2 was approached differently due to the dimness of the flash signatures and the relatively high proximal signal from moonlit clouds encountered during the scan (cloud signal was smeared due to the lack of motion compensation). The pixel array input to the algorithm contained all signal above the electronic offset level and the "readout" parameter was set to 13 to account for the highest locally observed pixel noise in the window. The background level determined during the thresholding stage was subtracted after de-convolution. Only "string of pearls" Flash 1 had sufficient intensity to be de-convolved for the purpose of assessing its shape. Uneven cloud signal close to the remaining dimmer flashes prevented a reliable de-convolution of their shapes.

Flash duration. The minimum resolvable flash duration at Juno's 2-revolutions per second spin rate is $2.7 \mathrm{~ms}$. When the SRU is operated without motion compensation (NoTDI mode) the scene is smeared along the CCD column direction at a rate of one pixel every $2.7 \mathrm{~ms} .{ }^{14}$ Therefore, a flash with duration $\leq 2.7 \mathrm{~ms}$ would have an along-column dimension of only 1 pixel following de-convolution. Each additional pixel of along-column smear adds $2.7 \mathrm{~ms}$ to the estimated maximum duration. The number of rows of pixels between each flash indicates the inter-flash time separation. Extended Data Fig. 7 illustrates maximum duration for the three NoTDI flashes that could be de-convolved. The true profile of "string of pearls" Flash 1 had an along-column size of two pixels (and an along-row size of 1 pixel). Because the two pixels have similar intensities and encompass the entire flash, we suspect that the center of the flash resides near the boundary of these two pixels and that the actual flash size is one pixel or smaller. The actual duration may therefore be shorter than $5.4 \mathrm{~ms}$. Another possibility, not resolvable by the SRU, is that all NoTDI flashes have a duration $<2.7 \mathrm{~ms}$ and the flash measurements represent the true dimensions without any image smear. Maximum dimension estimates for this assumption are shown as the second entry for each NoTDI flash in Extended Data Table 1.

When the SRU is operated with motion compensation (TDI mode) the rows of the image are shifted towards an opaque storage region at a rate of one row every $2.7 \mathrm{~ms} .{ }^{14}$ The first row of 
useable data (numbered 1 in Supplementary Data 2) experiences an effective exposure time of only $8.1 \mathrm{~ms}$ (two "dummy" rows of unexposed pixels precede this row). The flash duration resolution is computed by determining the boundary of the flash signature that is closest to the storage region and multiplying $2.7 \mathrm{~ms}$ by a factor equal to its row coordinate, plus two. The result is the total time this row of the image was exposed to the scene, the total time the storm was detectable by the SRU. When this computed value exceeds the commanded exposure time (for example, flashes from $1 \mathrm{~s}$ exposures with boundaries above row 368 ) the exposure time is the resolvable flash duration. These are the "effective exposure times" shown in Extended Data Table 1. As with the NoTDI flash signatures, the durations of the flashes collected in TDI mode may have been much shorter than the effective exposure time.

Estimates of flash size. The maximum dimension of each flash was estimated based on visual inspection of the deconvolution solution. We assessed the maximum number of pixels which could be assumed fully illuminated by flash photons, given that SRU flashes are small and not fully spatially resolved. The longest dimension of this patch in pixel units was multiplied by the optical distortion-corrected resolution (in $\mathrm{km}$ ). Because the SRU was not pointed normal to the Jovian cloud surfaces where the flashes were observed, we then corrected for foreshortening due to the emission angle. The flash width was multiplied by the secant of the appropriate emission angle component as calculated in the SRU frame (along-row or along-column, depending on flash orientation in the image plane). A second estimate was made for flashes observed in NoTDI images. Here we assumed a smaller steady state flash was smeared along the scan direction during the exposure (see Extended Data Fig. 7). Additionally, each NoTDI estimate was performed for the mapping of the flash at both the start and end of the exposure, due to the position uncertainty created by the scan and the resulting effect on pixel resolution. A similar approach was used for the HWHM results discussed in the text.

We assessed potential transverse image smear due to Juno's high linear velocity relative to Jupiter (>40 km/s for northern hemisphere observations, and $\sim 31 \mathrm{~km} / \mathrm{s}$ for the southern hemisphere observation) by computing the distance between the mapped flash locations at the beginning and end of the effective exposure time. This transverse distance was mapped into the image plane in along-row and along-column components and was typically found to be negligible (much smaller than a pixel). The four flashes with the longest effective exposure times 
423 (750 ms to $1 \mathrm{~s}$ ) had computed components that ranged from $0.32-1.06$ units of pixel smear.

424 However, given the very short flash durations observed in NoTDI imagery, we did not

425 incorporate these worst case smear factors into our flash size estimates.

426 Flash optical energy. The optical energy of each flash was estimated following the method used 427 by the Galileo lightning investigators. ${ }^{1}$

$$
\text { Flash Energy }=\frac{(\text { DN Sum })(\text { Pixel Area }) 2 \pi(\text { bandwidth })}{(\text { camera sensitivity })(\text { vingetting })\left(\cos ^{4}\right)}
$$

DNsum is the background-subtracted signal from all pixels in the flash signature. The Pixel Area was computed by multiplying the along-row pixel size by the along-column pixel size at the centroid location (following corrections for optical distortion). The SRU bandwidth is $650 \mathrm{~nm}$.

432 Field-dependent energy corrections for vignetting and $\cos ^{4}$ law losses ${ }^{36}$ were made to the energy estimates. Vignetting correction factors were based on observations of relative signal losses in SRU images collected without motion compensation where stars appeared as along-column streaks and showed decreased signal levels towards the edges of the field of view. Supplementary Data 4 provides the star streak data. The along-row and along-column components of each flash centroid were matched to star streak data with similar coordinates in order to estimate the potential field-dependent signal reduction. For optical systems such as the SRU there is a slight fall off of illumination in the image plane in the direction heading away from the optical axis. We model this loss using the "cosine fourth law," where the signal is

442 Flash energies were divided by vignetting correction factors ranging from $0.75-1$ and $\cos ^{4}$ factors ranging from 0.94-0.99, shown in Extended Data Table 2.

Because SRU lightning flashes are small and not fully spatially resolved we performed a second energy estimate treating the flash as a point source which emits over $4 \pi \mathrm{sr}$.

$$
\text { Energy }(\text { point source })=\frac{(\text { Energy outside }) 4 \pi}{(\Omega)(\text { vignetting })\left(\cos ^{4}\right)}
$$

447 where the vignetting and $\cos ^{4}$ correction factors are as noted above. "Energy outside" is the 448 energy of the flash that reached the SRU optics

$$
\text { Energy outside }=(\mathrm{DNsum})\left(2.6 \times 10^{-17} \mathrm{~J} / \mathrm{DN}\right)
$$


and $\Omega$ is the solid angle subtended by the active collection area of the SRU optics as seen from the flash

$$
\Omega=\frac{A_{o}}{\left(\text { Range } \times 10^{5}\right)^{2}}
$$

Range is the range of Juno to the flash in kilometers (calculated to the 1 bar level using an oblate spheroid Jupiter shape model).

For flashes acquired in NoTDI mode, the optical energy values reported herein are the average of the value computed at the start of the exposure and the value computed at the end of the exposure.

Global flash rate estimate. A lower bound on the global flash rate can be computed by using both NoTDI image scans to determine the average flash rate per storm. The approach is similar to the method used for the Galileo global flash rate estimate, ${ }^{1}$ but it utilizes two scanned observations. We make the conservative assumption that the "string of pearls" and "neighbor" flashes came from two separate storms and that both storms were visible for the full $1 \mathrm{~s} \mathrm{scan}$. Although there is a range of possible storm locations that would not have been visible for the entire $1 \mathrm{~s}$ exposure, this assumption will produce the minimum flash rate per storm. If we count 5 flashes in NoTDI Image \#2, conservatively treating "string of pearls" Flash 3 as a single event, the result is 2.5 flashes storm ${ }^{-1} \mathrm{~s}^{-1}$. A similar exposure time assumption for the two single-flash storms observed in NoTDI Image \#1 (with maximum durations of $8.1 \mathrm{~ms}$ and $16.2 \mathrm{~ms}$ ) yields a rate of 1 flash storm ${ }^{-1} \mathrm{~s}^{-1}$. The average rate is 1.75 flashes storm ${ }^{-1} \mathrm{~s}^{-1}$. Multiplying by 11 observed storms and dividing by the total surveyed area of $1 \times 10^{10} \mathrm{~km}^{2}$, the global flash rate is $6.1 \times 10^{-2}$ flashes $\mathrm{km}^{-2} \mathrm{yr}^{-1}$. This rate represents an average value and is not meant to imply that lightning is equally likely to occur at any location in Jupiter's atmosphere. For comparison, the average annual global flash rate on Earth is $\sim 3$ flashes $\mathrm{km}^{-2} \mathrm{yr}^{-1} .37$

Some SRU images were not fully downlinked from the spacecraft due to imaging cadence constraints and some contained portions of dark sky. When this was the case, only the projected areas of pixels containing Jupiter were counted in the surveyed area. Pixels that were not downlinked are shown with zero values in Supplementary Data 2. The two dummy columns and 
477 dummy rows are also represented with zero pixel values. The dummy rows are placed in the 511

478 and 512 row positions, which is an artifact of Juno's data de-commutation software.

479 MWR lightning detection filtering criteria. The MWR lightning detections recorded in

480 Supplementary Data 1 were selected based on the following criteria: the MWR antenna gain at

481 the projected location of the SRU flash detection was greater than -20dB relative to the peak

482 antenna gain, the time difference between the MWR detection and the start of the SRU exposure

483 was less than $185 \mathrm{~s}$, and the MWR-detected lightning power was greater than three standard

484 deviations above the noise floor.

485 Lightning generation above the 2 bar level. We present two considerations for charge 486 separation in the 1-2 bar region of Jupiter's atmosphere.

487 In simulations of cloud formation at Jupiter ${ }^{21}$ small water ice crystals can be lofted to the 1 bar 488 region, but the sticking efficiency of ice particles is small relative to liquid. Hence, in a purely 489 icy regime, the efficiency of particle growth, charge separation, and lightning generation may be 490 less efficient, unless dynamical circumstances enhance the spread of particle sizes, such as in 491 anvils (discussed below). While liquid water is not expected at altitudes higher than the $\sim 3$ bar 492 level (as temperatures there fall below the $\sim-40^{\circ} \mathrm{C}$ limit for supercooled liquid water), the 493 freezing point of water should not define the altitude limit of liquid droplets in Jovian clouds. 494 The equilibrium thermodynamics of the $\mathrm{NH}_{3}-\mathrm{H}_{2} \mathrm{O}$ mixture ${ }^{22,23}$ shows that for a volume mixing of 495 ammonia of 200 to 360 ppmv, as measured at Jupiter by Juno's microwave radiometer, ${ }^{29,30}$ 496 ammonia tends to be adsorbed into water ice crystals to form a liquid $\mathrm{NH}_{3}-\mathrm{H}_{2} \mathrm{O}$ mixture at 497 temperatures between $-85{ }^{\circ} \mathrm{C}$ and $-100{ }^{\circ} \mathrm{C} .{ }^{24}$ Hence these $\mathrm{NH}_{3}-\mathrm{H}_{2} \mathrm{O}$ droplets would be created 498 499 500 501 502 between $\sim 1.1$ and 1.5 bars, just above the 1.4 to 1.9 bar region where Juno's observations of shallow lightning originate. As the droplets fall into the shallow lightning region they can collide with water-ice particles that are still moving upward in the updraft, or even with water-ice particles that are falling with a smaller terminal velocity than the droplets. Both the number of particles and their terminal velocity differences would increase. The subsequent movement of the particles away from each other would separate charge. Thus, this extension of the liquid field provides a concomitant enlargement of the pressure-temperature regime over which charge exchange, growth of particles, and hence lightning may occur. 
506

507

508

509

510

511

512

513

514

515

516

517

518

519

520

521

522

523

524

525

526

527

528

529

530

531

532

533

Alternatively, lightning generation by charge exchange between solid icy particles is also possible, because of additional effects in the microphysics of the collisions and potential large scale dynamical effects. With regard to the former, additional effects that have been identified include the enhancement of charge buildup by ionic impurities, and the formation of transient liquid interfaces during ice-ice collisions. ${ }^{38}$ With regard to the latter, lightning generation may be enhanced in anvil clouds. Anvils are special environments in mature thunderstorms in which strong horizontal winds and a transition from upwelling to downwelling flow are present; hence a large range of particle sizes can be found there. A similar environment may be responsible for Juno's observations of shallow lightning (we give credit to one of the referees for bringing this option to our attention). Intense storms have been observed to reach above the 1 bar level and have been attributed to deep rooted moist-convective water storms that can pierce Jupiter's ammonium hydrosulfide and ammonia cloud decks. ${ }^{12}$ Additionally, modeling has shown that updrafts in Jovian convective clouds can transport condensates at velocities of several tens of meters per second, allowing water ice particles of different sizes to develop and be lofted up to these altitudes. ${ }^{21,39,40}$ Therefore ice-ice collisions in Jovian anvils ${ }^{28}$ or high reaching moistconvective water storms are plausible paths to lightning generation.

\section{References}

32. Klaasen, K.P, et al. Inflight performance characteristics, calibration, and utilization of the Galileo solid-state imaging camera. Opt. Eng. 36(11), 3001-3027 (1997).

33. NASA NAIF website. https://naif.jpl.nasa.gov/pub/naif/JUNO/kernels/ (2019).

34. Acton, C.H. Ancillary data services of NASA's Navigation and Ancillary Information Facility;" Planetary and Space Science, 44 (1), 65-70 (1996).

35. Deconvlucy, https://www.mathworks.com/help/images/ref/deconvlucy.html (2019).

36. D.C. O’Shea, Elements of Modern Optical Design. (John Wiley and Sons, Inc., Chichester, 1985).

37. Christian, H.J., et al. Global frequency and distribution of lightning as observed from space by the Optical Transient Detector. J. Geophys. Res., 108(D1), 4005, doi:10.1029/2002JD002347 (2003). 
38. Nelson, J. \& Baker, M. Charging of ice-vapor interfaces: applications to thunderstorms. Atmos. Chem. Phys. 3, 1237-1252 (2003).

39. Yair, Y., Levin, Z., \& Tzivion, S. Model interpretation of Jovian lightning activity and the Galileo probe results. Journal of Geophysical Research 103, D12, 14,157-14,166 (1998).

40. Hueso, R., Sanchez-Lavega, A., and Guillot, T. A model for large-scale convective storms in Jupiter. Journal of Geophysical Research 107, E10, 5075, doi:10.1029/2001JE001839 (2002).

Data availability. The authors declare that the Juno SRU data supporting the findings of this study are available within the paper and its supplementary information files. The Juno MWR data that support the findings of this study are available from the Planetary Data System archive (https://pds.nasa.gov/index.shtml) as 'Juno Jupiter MWR reduced data records v1.0' (dataset JNO-J-MWR-3-RDR-V1.0). Source data for Figure 2 and Extended Data Figure 1 are provided with the paper.

Acknowledgements The authors thank Gianni Berrighi and Simone Becucci of the Leonardo Finmeccanica S.p.A. (formerly Selex Galileo S.p.A) Juno SRU Team for retrieval of SRU optics and CCD quantum efficiency parameters used in the study. J.E.P. Connerney is thanked for comments on the manuscript. J. Arballo is thanked for rendering of figures and tables. M. Stetson is thanked for artistic rendering of Figure 3. This research was carried out at the Jet Propulsion Laboratory, California Institute of Technology, under a contract with the National Aeronautics and Space Administration; at the Observatoire de la Côte d'Azur under the sponsorship of the Centre National d'Etudes Spatiales; and at the Southwest Research Institute under contract with NASA. Copyright 2020. All rights reserved.

Author Contributions H.N.B. led the acquisition and interpretation of SRU lightning data, wrote the manuscript with input from coauthors, and performed the SRU camera response computations. S.Bo. and T.G. contributed to the interpretation of shallow lightning atmospheric dynamics. M.B. contributed to the acquisition of SRU lighting data and performed the SRU observation geometry computations. J.A. contributed to SRU camera response computations, flash identification and mapping, and analysis of camera vignetting characteristics. A.G. computed the SRU survey area. S.A. and P.S. contributed expertise in Jovian atmospheric dynamics and composition. J.L. assisted with the ammonia-water thermodynamics, the lightning 
563 generation discussion, and construction of Figure 3. Y.A. contributed to the lightning generation

564 discussion. A.I. contributed to the SRU data interpretation. S.Br. analyzed the MWR data to

565 extract and filter MWR lighting observations. S.L. is the lead of the MWR.

566 Competing Interests The authors declare no competing interests.

567 Supplementary Information is available for this paper.

568 Correspondence and requests for materials should be addressed to Heidi.N.Becker@jpl.nasa.gov.

569 Reprints and permissions information is available at www.nature.com/reprints.

570

571

572

573

574

575

576

577

578

579

580

581

582

583

584

585

586

587

\section{Extended Data Table 1 | Record of lightning flashes observed by the Juno SRU}

Flash names are interpreted Perijove Number_Image Number_Flash ID Number. 14_12 is

NoTDI image \#1 and 17_13 is NoTDI image \#2 (see text and Methods). TDI denotes motion compensation by time delay integration. Flashes observed without motion compensation in NoTDI mode have entries which correspond to mappings at the start of the image exposure (first entry) and at the end of the image exposure (second entry, with asterisk). The "Effective Exposure" is the maximum time the flash location was observed by the SRU. Ranges are from the Juno spacecraft to the flash location on Jupiter (at the 1 bar level). Longitudes are System III West longitudes. HWHM is the half-width at half maximum intensity distance. Energies in the second column from the right assume each flash is a patch of light on a Lambertian surface. Energies in the rightmost column treat the flash as a point source.

\section{Extended Data Table 2 | Supplemental parameters used in flash mapping and energy calculations}

Time tags correspond to the start of the SRU image exposure. Row and column coordinates of calculated flash centroids, cos4 correction factors, and vignetting correction factors are shown. Flash names are as in Extended Data Table 1. 
Extended Data Fig. 1 | Properties of the SRU optical system. a. Energy distribution of the camera's point spread function, shown for an image of a point source. The scale bar indicates the percentage of the total signal. b. The combined throughput of the SRU optical system, QT (CCD quantum efficiency "QE" $\times$ optics transmission “T”), as a function of wavelength.

\section{Extended Data Fig. 2 | Overlap of MWR Antenna 1 beam and SRU field of view during} lightning detections. Circular 17-degree MWR beam contours (green and red circles) for MWR lightning detections acquired within 30 seconds of an example SRU lightning flash detection (inside the yellow circle). Red MWR beam contours correspond to footprint locations during the 1 s SRU image exposure (start time 2018-144T04:57:50.263).

\section{Extended Data Fig. 3 | Estimated half-width at half-maximum values. De-convolved}

lightning flash signatures are shown for flashes where a HWHM could be estimated. Estimates represent the maximum possible value. The white circle indicates the maximum pixel area which can be assumed fully illuminated by flash photons given spatial resolution limitations. The estimated HWHM was generally less than the size of one pixel width. The red line in Flash 15_13_1 (panel e) indicates the diagonal distance of the estimated HWHM.

\section{Extended Data Fig. 4 | Morphology of signatures from optical vs. ionizing radiation} sources. SRU Image 12, Perijove 13. Insets show magnified views of example signatures from an optical source (lightning, circled in yellow), and an ionizing radiation source (penetrating particle, circled in blue). Dimmer pixels are blue and brighter pixels are yellow. Signatures from optical sources have a more symmetric appearance which follows the camera point spread function.

Extended Data Fig. 5 | SRU pixel coordinate system conversion. Illustration of the transformation from a pixel array numbered 1 to 512, to the 0 to 511 pixel coordinate system of the SRU instrument frame. 
618 Extended Data Fig. 6 | Reconstruction of flash true profile. Example reconstruction for SRU

619 lightning flash 11_12_1. a. Observed flash signature. b. Deconvolution solution; estimated flash 620 shape on Jupiter. The white circle indicates the maximum pixel area which can be assumed fully 621 illuminated by flash photons given spatial resolution limitations. 1-2 pixels are estimated to be 622 fully illuminated in this example. c. Result following convolution of the estimated shape with the 623 camera point spread function. d. Residual signal (a. minus c.).

624

625 Extended Data Fig. 7| Maximum durations of flashes observed with NoTDI. a. Deconvolved 626 SRU flash 14_12_15. The white circles indicate a possible flash area for a steady state source at 627 the start (lower) and end (upper) of the exposure. The spacecraft spin direction and the direction 628 in which the scene will smear are indicated with white arrows. The maximum possible duration 629 is $8.1 \mathrm{~ms}(\sim 3$ rows of smear along-column). b. same for SRU flash 14_12_17; maximum flash 630 duration 10.8 to 16.2 ms. c. same for SRU flash 17_13_4 ("string of pearls" Flash 1); maximum 631 duration $5.4 \mathrm{~ms}$. 